\title{
SIMULATION ACCURACY IN RADIOTHERAPY FOR MAXILLARY SINUS TUMORS
}

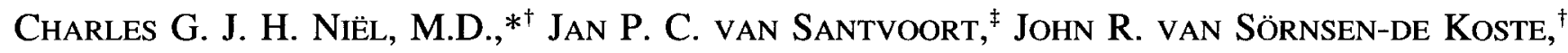 \\ Peter J. C. M. NowaK, M.D. ${ }^{\dagger}$ And Peter C. Levendag, M.D., Ph.D. ${ }^{\dagger}$ \\ Departments of ${ }^{\dagger}$ Radiation Oncology and ${ }^{\ddagger}$ Clinical Physics, Dr. Daniel den Hoed Cancer Center, Rotterdam, The Netherlands
}

\begin{abstract}
Purpose: To evaluate the accuracy and clinical importance of beam positioning during simulation of radiation treatment for tumors in the maxillary sinus.

Methods and Materials: Five patients were prepared as if they were to be treated for a maxillary sinus tumor. A three-beam computed tomography (CT) scan-based computer plan was made for each patient. The location of the central beam axis of each beam was measured, relative to bony anatomical structures. A simulation was performed using the bony references to position the radiation beams during simulation. After this, the simulation procedure was repeated by the use of a noninvasive external localization frame with a known accuracy and reproducibility within $2 \mathrm{~mm}$ margins.

Results: When defining the clinical target volume as the known tumor with a $1 \mathrm{~cm}$ margin, three out of five patients would suffer a partial geographical miss throughout the entire radiation treatment due to erroneous beam positioning at the simulation stage when using bony structures as a guide for beam positioning. The influence of these errors is analyzed as normal tissue complication and tumor control probabilities.

Conclusion: When defining a planning target volume, one should consider a margin to correct for possible simulation errors. We advise the use of objective, external (and thus nonanatomical) landmarks as a reference during simulation to reduce this extra margin to a minimum. In case of simulation, using bony structures as a reference, an additional margin should be entered, depending on the simulation accuracy that can be obtained.
\end{abstract}

Maxillary sinus cancer, Radiotherapy, Treatment simulation, Accuracy, Local control, Complication.

\section{INTRODUCTION}

One of the major advances in clinical radiotherapy during the last 10 years has been the introduction of conformal beam therapy. This treatment modality requires a high precision in both preparation and the actual treatment stage. The complexity of radiation therapy for maxillary sinus tumors resembles conformal beam therapy to a certain extent: covering the target volume is largely restricted by the tumor-surrounding normal tissues (14). Moreover, beam positioning during treatment simulation is hampered by the large amount of bony structures that can serve as a landmark, easily leading to a situation in which one cannot see the wood for the trees. Lack of accuracy in radiation treatment preparation for maxillary sinus tumors might therefore be a reason why these tumors recur in a large number of patients (up to $80 \%$ of the patients are faced with local recurrence) (19), even after a complete remission after radiotherapy alone or in combination with surgery or regional chemotherapy (the so-called Sato's treatment) (20). Indications for the validity of this statement are publications on the influence of the introduction of computed tomography (CT) scanning in radiotherapy planning of maxillary sinus tumors, after which the local control rate and the survival of patients with a maxillary sinus tumor increased significantly $(8,9,18,22)$.

The high number of unexpected radiotherapyrelated complications to tumor-surrounding normal
* Present address: Westeinde Hospital, Dept. of Radiotherapy, Lijnbaan 32, 2512 VA, The Hague, The Netherlands.

Reprint requests to: C. G. J. H. Niël, M.D., Dr. Daniel den Hoed Cancer Center, Dept. of Radiation Oncology, P.O. Box 5201, 3008 AE Rotterdam, The Netherlands.
Acknowledgements - The authors gratefully acknowledge Ms. Inge Dijkstra for assistance in preparing the manuscript and Dick van Sluis for his never-ending patience in improving the noninvasive external localization frame.

Accepted for publication 19 August 1994. 


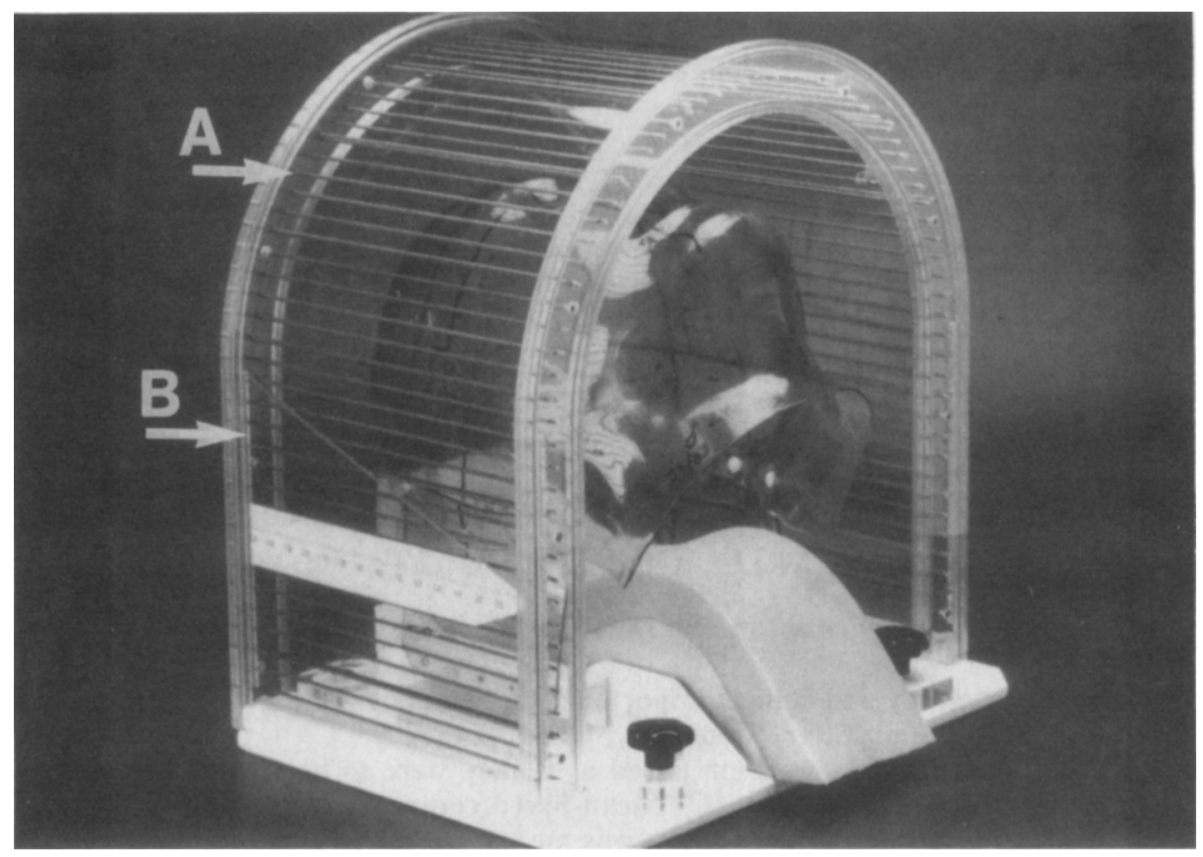

Fig. 1. Noninvasive external localization frame with patient fixed in position by a vacuum mask. (A) parallel rods; (B) lucite triangle.

tissues is a second argument to consider radiation treatment preparation for maxillary sinus tumors suboptimal. We therefore tried to evaluate the beam positioning accuracy during treatment simulation, one of the most critical steps in radiotherapy, because an error made at this stage will be repeated throughout the entire treatment, largely influencing treatment outcome.

\section{METHODS AND MATERIALS}

For the aim of the study, a noninvasive external localization frame was developed (16). This frame was used to compare beam positions as a result of simulation, based on bony anatomical landmarks with beam positioning based on objective, external (and thus nonanatomical) numeric references.

The design of the frame is based on a concept similar to stereotactic frames used routinely in neurosurgery. In contrast to these frames, localization can be performed without computed calculation of coordinates by direct read-out of external numeric references.

The technique of this frame is described elsewhere (16). In brief, it consists of parallel graphite rods, located in the craniocaudal axis and parallel to the table top (Fig. 1). These rods are depicted on the CT scan as points, serving as a reference in the anteroposterior and laterolateral direction (Fig. 2). At one side of this frame a lucite triangle serves as a reference in the craniocaudal direction; this triangle is seen on the CT scan as a line with a certain length, depending on the location in the craniocaudal direction in which the CT scan is performed (Figs. $1 \mathrm{~b}$ and $2 \mathrm{~b}$ ). The accuracy and the clinical reproducibility of beam positioning using this frame was found to be within $2 \mathrm{~mm}$ margins (16).

Five patients were prepared as if they were treated for a maxillary sinus tumor. During each step of the procedure the patient was immobilized with a vacuum mask to assure reproducible patient positioning in the best achievable way (this is within $2 \mathrm{~mm}$ margins under optimal conditions $(6,22,25)$. CT planning was performed for a three-beam radiation technique, the planning target volume being confined to the maxillary sinus, the tuber maxillae, and the bottom of the orbita, all with a $1 \mathrm{~cm}$ margin to cover subclinical disease. CT scans with the noninvasive external localization frame were performed every $5 \mathrm{~mm} .{ }^{1}$ A three-dimensional (3D) treatment plan was made with the Cadplan treatment planning system. ${ }^{2}$ One anterior and two lateral beams were chosen to adequately cover the target volume, and beam blocks were positioned in the beam's eye view to reduce the dose of the surrounding normal tissues to a minimum. This resulted in a homogeneous irradiation of the target volume to a total dose of $70 \mathrm{~Gy}$, with a maximum dose of $20 \%$ to the contralateral lens of the

\footnotetext{
${ }^{1}$ Siemens Somatom Plus S, Siemens, The Hague, The Netherlands.
}

\footnotetext{
${ }^{2}$ Dosetek-Varian, Espoo, Finland.
} 


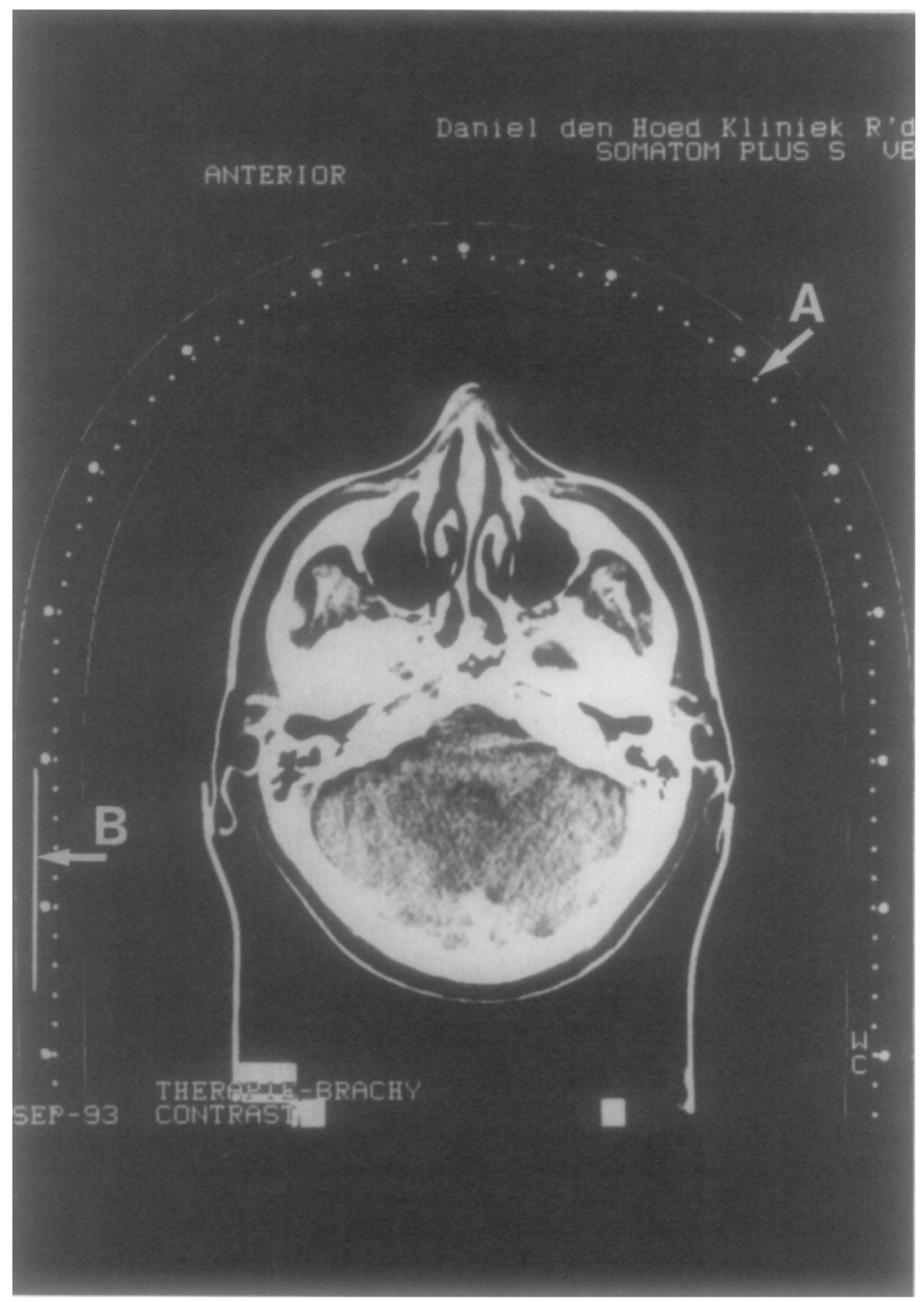

Fig. 2. CT-scan image of immobilized patient with the noninvasive external localization frame. (A) Parallel rods depicted as dots; (B) lucite triangle depicted as a line.

eye and of $64 \%$ to the spinal cord (Figs. $3 a$ and $3 b$ ). Each patient was simulated according to his CT-based treatment plan, using the bony structures as a reference for beam positioning during simulation. At this stage the beam portal outlines were indicated on the patient to enable reproducible beam positioning throughout the entire treatment. To avoid introduction of a bias, the simulations were performed by radiation oncologists other than the authors. The noninvasive external localization frame was used to check the accuracy of the simulations. The advantage of this system over the use of radio-opaque catheters mounted on a patient's mask (24) is its ease in use and the addition of references in the craniocaudal direction.

The difference between beam positions, resulting from treatment simulation using bony references and beam positions as achieved by using the noninvasive external localization frame, was noted in the laterolat- eral and craniocaudal direction for the anterior portal, and in the anteroposterior and craniocaudal direction for the right and left lateral portal (all expressed in $\mathrm{mm}$ ). New dose distributions were calculated, applying these beam positioning errors on the computer plan of one patient, serving as the reference plan.

A dose volume histogram (DVH) analysis of these dose distributions was performed, together with an analysis of normal tissue complication probability (NTCP) and tumor control probability (TCP) values. The TCPs were calculated from the DVHs according to the clinical response model of Goitein (5), also described by Munzenrider (15). The parameters (doseresponse curve for maxillary sinus tumors) for this model were obtained from the data from the literature $(2,11,13)$. The NTCPs were calculated according to the model by Kutcher (12), using the data of Emami (3), and fitted as described by Burman (1). 


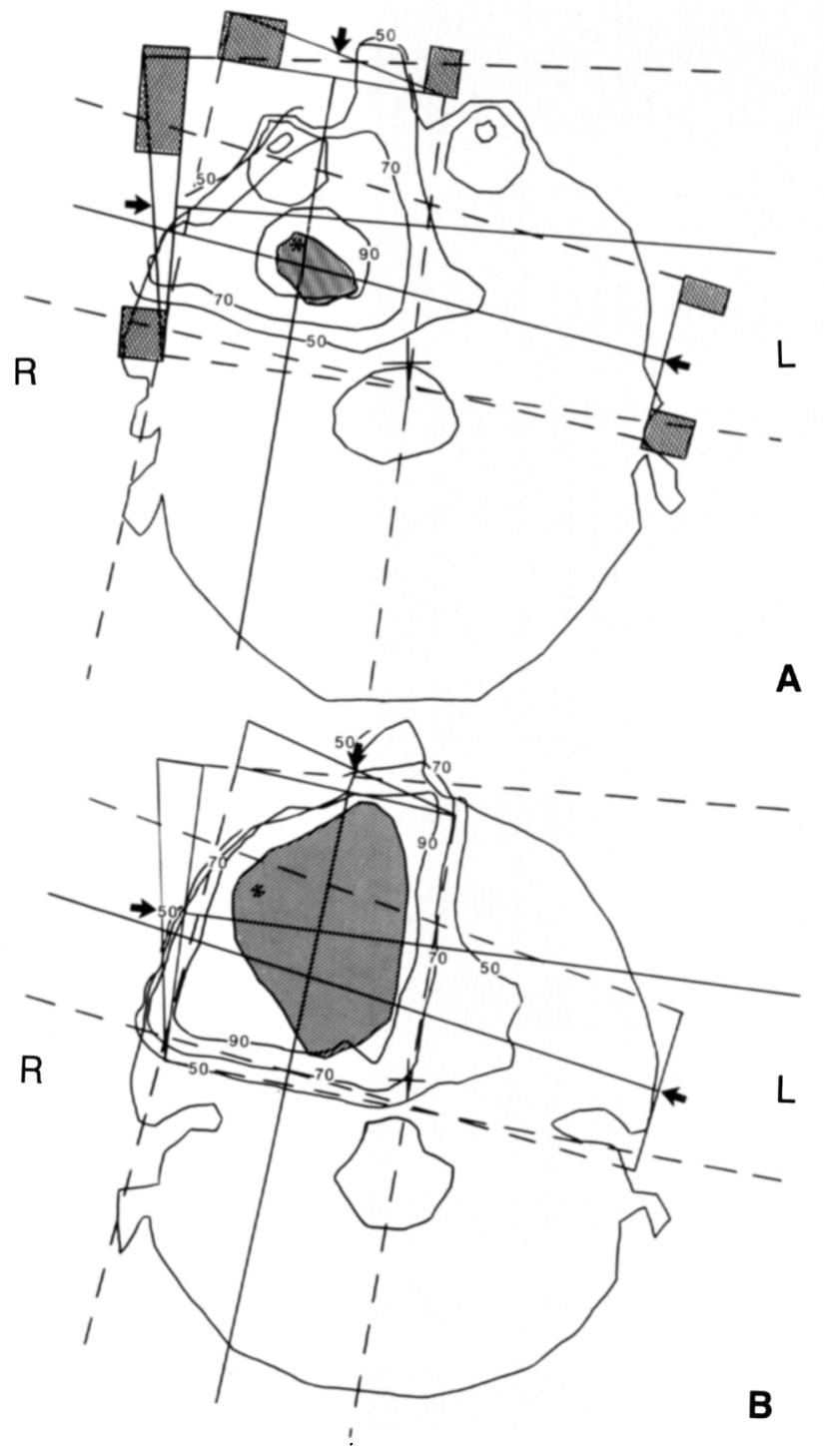

Fig. 3. (A) Three-beam computer-assessed 3D treatment plan (Cadplan, Dosetek-Varian); plane through eye lens (70 Gy prescribed dose at $90 \%$, normalization according to ICRU). (B) Three-beam computer-assessed 3D treatment plan (Cadplan, Dosetek-Varian); central plane (70 Gy prescribed dose at $90 \%$, normalization according to ICRU).

\section{RESULTS}

The beam positioning errors made during simulation for maxillary sinus tumors are given for each patient and as an average in Table 1. Although the mean error made for all patients is low, indicating that these errors occur at random, the individual dislocation of the beams was unexpectedly large. In three out of the five patients (patients 1,3 , and 5) it was found to be more than $1 \mathrm{~cm}$ for at least one beam in at least one direction. This also becomes clear from the standard deviation for the errors in the three directions. Because the positioning error is introduced during simulation, the greatest error is the single absolute greatest error in whatever direction and not the mean error per patient in the three directions. It is then clear that three out of five patients will have a systematic error (in at least one direction) introduced of at least $1 \mathrm{~cm}$. These patients would therefore suffer a partial geographical miss throughout the entire treatment when defining the clinical target volume as the known tumor with a $1 \mathrm{~cm}$ margin.

The possible clinical value of enhancing simulation accuracy in maxillary sinus tumors was assessed by the DVH, TCP, and NTCP analysis. All five patients were compared with the computer-assessed plan serving as the best achievable clinical result (the intended plan). In ideal circumstances, that is, treatment simulation identical to the computer plan (which can be achieved within $2 \mathrm{~mm}$ accuracy by the use of the noninvasive external localization frame), the DVH shows a very steep fall-off from $100 \%$ to $0 \%$ at $70 \mathrm{~Gy}$ (Fig. 4). The patients in whom simulation was performed using bony landmarks as a reference for beam positioning clearly suffer from a partial geographical miss, as can be seen in their respective target DVH (Fig. 4). As expected, the patient with the largest simulation error has the lowest TCP. As an average, the TCP for the five patients was about one-third $(27 \%)$ of the TCP of the intended plan (78\%, Table 2).

As to the normal tissues, the contralateral eye lens, when irradiated according to the treatment plan, receives a dose well below its tolerance, which also holds for those patients with the smallest simulation errors. However, in the two patients with the largest beam positioning errors, the contralateral eye lens is irradiated to a considerably higher dose, which is expressed in a large NTCP value (Table 2).

In contrast, the right eye lens (which receives the full dose when irradiating according to the computer plan) was found to receive doses well below its tolerance level in the patients with the largest beam positioning errors (Table 2). The spinal cord in all cases was irradiated to a dose well below its tolerance due to the larger distance to the target volume (Table 2).

Table 1. Beam position errors at time of stimulation, detected by the noninvasive external localization frame

\begin{tabular}{|c|c|c|c|c|c|c|}
\hline \multirow{2}{*}{ 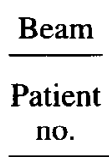 } & \multicolumn{2}{|c|}{ Left } & \multicolumn{2}{|c|}{ Right } & \multicolumn{2}{|c|}{ Anterior } \\
\hline & $\mathrm{CC}$ & AP & $\mathrm{CC}$ & AP & $\mathrm{CC}$ & AP \\
\hline 1 & -8 & 7 & -8 & 16 & -8 & 3 \\
\hline 2 & -10 & 6 & -10 & -8 & -10 & 10 \\
\hline 3 & 14 & 3 & 14 & 0 & 14 & 11 \\
\hline 4 & -10 & 5 & -10 & -2 & -10 & 0 \\
\hline 5 & 13 & -5 & 13 & 3 & 13 & 6 \\
\hline Mean & -0.2 & 3.2 & -0.2 & +1.8 & -0.2 & 6 \\
\hline $\mathrm{SD}^{*}$ & 11.2 & 4.3 & 11.2 & 8.0 & 11.2 & 4.1 \\
\hline
\end{tabular}

* $\mathrm{SD}=$ standard deviation $; \mathrm{CC}=$ craniocaudal, $\mathrm{AP}=$ anteroposterior; $\mathrm{LL}=$ laterolateral; all expressed in $\mathrm{mm}$. 


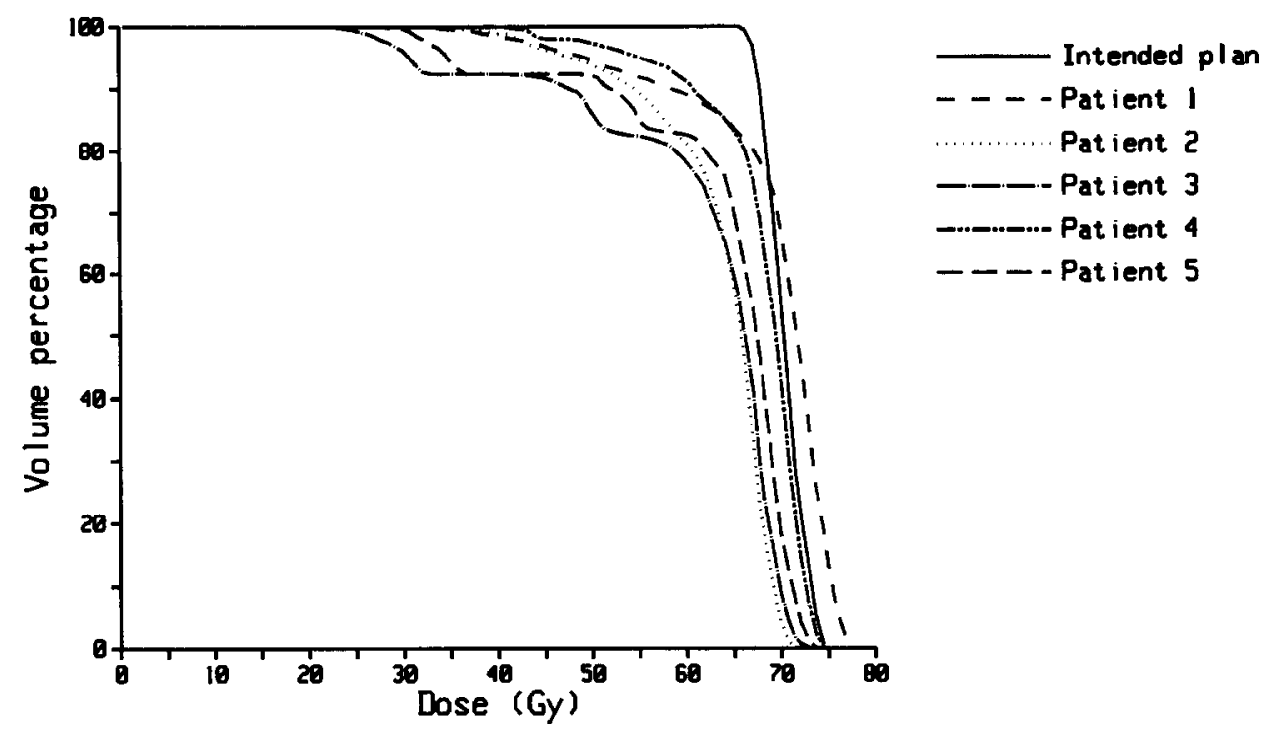

Fig. 4. Dose volume histogram for the target volume. Intended plan: simulated and treated according to treatment plan (noninvasive external localization frame). Patients $1-5$ : treated according to beam position errors of simulation (anatomical landmarks).

\section{DISCUSSION}

Much attention is given to the daily (random) beam positioning accuracy during radiation treatment. This uncertainty factor is accounted for in the planning target volume, defined as the clinical target volume with a margin necessary to avoid a partial geographical miss due to these daily beam or patient positioning errors (ICRU report no. 50). Virtually no information is found, however, about the magnitude and possible clinical relevance of errors made in the process of simulation when bony structures serve as a reference for beam positioning, a method still widely in use. Despite the considerable clinical influence of simulation errors, the reason for this lack of knowledge is unclear. The only publications dealing with the clinical importance of radiation treatment preparation analyze the influence of the type of treatment technique on treatment outcome, but do not address the problem of errors in the transfer from CT to simulator $(4,7,17)$.

Table 2. TCP- and NTCP values when simulated and treated according to treatment plan (intended plan: using the noninvasive external localization frame) or with simulation errors (patient 1-5: simulating by anatomical landmarks)

\begin{tabular}{lcccc}
\hline & TCP & $\begin{array}{c}\text { NTCP } \\
\text { ipsilateral } \\
\text { eye lens }\end{array}$ & $\begin{array}{c}\text { NTCP } \\
\text { contralateral } \\
\text { eye lens }\end{array}$ & $\begin{array}{c}\text { NTCP } \\
\text { spinal cord }\end{array}$ \\
\hline intended plan & 0.776 & 1.000 & 0.072 & 0.000 \\
patient no. 1 & 0.413 & 0.618 & 0.007 & 0.000 \\
patient no. 2 & 0.225 & 0.016 & 0.002 & 0.001 \\
patient no. 3 & 0.088 & 1.000 & 0.997 & 0.013 \\
patient no. 4 & 0.472 & 0.070 & 0.001 & 0.000 \\
patient no. 5 & 0.159 & 1.000 & 0.966 & 0.004 \\
mean (1-5) & 0.271 & & & \\
\hline
\end{tabular}

After constructing this "missing link" between a planning CT scan and localization x-ray film, we were able to evaluate the possible clinical importance of simulation errors. To study the influence on local control and side effects of potential beam positioning errors at the stage of simulation, we chose maxillary sinus tumors for a number of reasons. First, many radiation treatment techniques exist for these tumors, indicating the complexity of their irradiation. Second, these tumors have a high local recurrence rate, ranging between $20 \%$ and $70 \%$, and partly depending on the treatment technique used $(10,11,19)$. The highest local control rates are achieved by multimodality therapy in which high doses of radiation play an important role. Any improvement in radiation quality might therefore result in a better treatment outcome, as shown by Kondo and Tsujii $(8,9,23)$. They clearly showed that the introduction of CT scanning in the treatment of maxillary sinus tumors leads to higher local control and survival rates, probably due to a better target delineation. Finally, maxillary sinus tumors were used as a first test site because of the complexity of the treatment simulation procedure, due to the large amount of anatomical structures that may serve as a (mis)lead during simulation. We deliberately did not define an extra margin around the clinical target volume to correct for daily beam positioning errors, because we wanted to evaluate the influence of simulation errors only. This is in contrast to daily practice where a margin is chosen for daily beam and patient positioning errors without taking possible beam-positioning errors during simulation into account.

The results of our analysis are very much according to the clinical results of radiotherapy for maxillary sinus tumors. The beam positioning errors made during simulation were unexpectedly high and, in the DVH and TCP 
analysis, appear to be of great clinical importance. All patients suffer more or less from a partial geographical tumor miss due to simulation inaccuracy. This is reflected in the mean TCP value, which is about one-third of the maximal TCP $(78 \%)$ when simulating completely according to the computer-assessed plan. We do, however, like to stress that one cannot give any absolute value to these TCP data because little information exists on a clear dose effect on local control $(2,11,13)$, due to the enormous variation in treatment techniques. Our data show very clearly that simulation beam-positioning errors are of clinical importance and should be taken into account when defining a planning volume if anatomical landmarks are used during simulation. The additional margin that should be chosen for this reason will probably vary according to tumor site and the treatment technique used. For instance, a partial geographical miss due to simulation beam-positioning errors when using anatomical structures as a reference is very unlikely in $\mathrm{T} 1$ laryngeal cancer. In contrast, conformal beam therapy for a brain tumor with complex beam settings is more likely to give a significant partial geographical miss when using virtually absent anatomical landmarks as a simulation guide.

With regard to the normal tissues it became clear that the NTCP value of the spinal cord in none of the patients reached above the tolerance level. The contralateral eye lens, however, was shown to be irradiated to a level above tolerance in patients with the largest beam positioning errors. One might argue that these patients will not develop any lens complication due to a local tumor, which will certainly recur early because these patients were shown to have the largest geographical miss. Interest- ingly, the ipsilateral eye lens that was planned to be irradiated above its tolerance level (NTCP for the computer plan equalled 1), was apparently spared in two patients, again due to simulation beam-positioning errors. If one takes into account the higher probability for local recurrence after protecting the ipsilateral eye lens by a block $(2,21,23)$, one could seriously question the use of these lens blocks. As a result of our study, it can be concluded that these blocks should be positioned with the highest care, preferably checked daily on a clinical basis.

\section{CONCLUSIONS}

The planning target volume for maxillary sinus tumors should include an additional margin to correct for possiblc partial geographical misses due to simulation errors caused by the transfer from the CT to the simulator. This error is likely to be smallest when easy to use, objective, and external references (such as our noninvasive external localization frame) serve as a guide for beam positioning during simulation. If anatomical landmarks are used during the entire process of CT scanning, treatment planning, and simulation as a reference, this extra margin will considerably enlarge the treated planning target and thereby the treated volume. As a result, from our study, we advise that the use of anatomical landmarks during simulation be reduced to keep the margin around the clinical tumor volume (defined to account for set-up uncertaintics) at a magnitude such that surrounding critical structures can still be spared from high radiation doses.

The possible clinical relevance of simulation errors in tumor sites other than the maxillary sinus is the issue of investigations currently being undertaken at our institute.

\section{REFERENCES}

1. Burman, C.; Kutcher, G. J.; Emami, B.; Goitein, M. Fitting of normal tissue tolerance data to an analytic function. Int. J. Radiat. Oncol. Biol. Phys. 21:123-135; 1991.

2. Bush, S. E.; Bagshaw, M. A. Carcinoma of the paranasal sinuses. Cancer 50:154-158; 1982.

3. Emami, B.; Lyman, J.; Brown, A.; Coia, L. R.; Goitein, M.; Munzenrider, J. E.; Shank, B.; Solin, L. J.; Wesson, M. Tolerance of normal tissue to therapeutic irradiation. Int. J. Radiat. Oncol. Biol. Phys. 21:109-122; 1991.

4. Gaspar, L. E.; Fisher, B. J.; Macdonald, D. R.; LeBer, D. V.; Halperin, E. C.; Schold, S.C., Jr.; Cairncross, J. G. Supratentorial malignant glioma: Patterns of recurrence and implications for external beam local treatment. Int. J. Radiat. Oncol. Biol. Phys. 24:55-57; 1992.

5. Goitein, M. The probability of controlling an inhomogeneously irradiated tumour. In: Zink, S., ed. Evaluation of Treatment Planning for Particle Beam Radiotherapy. Bethesda: National Cancer Institute; 1987:1-13.

6. Huizenga, H.; Levendag, P. C.; De Porre, P. M. Z. R.; Visser, A. G. Accuracy in radiation field alignment in head and neck cancer: A prospective study. Radiother. Oncol. $11: 181-187 ; 1988$.

7. Kinzie, J. J.; Hanks, G. E.; Maclean, C. J.; Kramer, S. Patterns of care study: Hodgkin's disease relapse rates and adequacy of portals. Cancer 52:2223-2226; 1983.
8. Kondo, M.; Horiuchi, M.; Inuyama, Y.; Dokiya, T.; Tsutsui, T.; Iwata, Y.; Endo, M.; Hashimoto, T.; Kunieda, E.; Hashimoto, $S$. Value of computed tomography for radiation therapy of tumors of the nasal cavity and paranasal sinuses. Acta Radiol. Oncol. 22(fasc. 1):3-8; 1983.

9. Kondo, M.; Horiuchi, M.; Shiga, H.; Inuyama, Y.; Dokiya, T.; Takata, Y.; Yamashita, S.; Ido, K.; Ando, Y.; Iwata, Y.; Hashimoto, S. Computed tomography of malignant tumors of the nasal cavity and paranasal sinuses. Cancer 50:226-231; 1982.

10. Kondo, M.; Ogawa, K.; Inuyama, Y.; Yamashita, S.; Tominaga, S.; Shigematsu, N.; Nishiguchi, I.; Hashimoto, S. Prognostic factors influencing relapse of squamous cell carcinoma of the maxillary sinus. Cancer 55:190-196; 1985.

11. Korzeniowski, S.; Reinfuss, M.; Skolyszewski, J. The evaluation of radiotherapy after incomplete surgery in patients with carcinoma of the maxillary sinus. Int. J. Radiat. Oncol. Biol. Phys. 11:505-510; 1985.

12. Kutcher, G. J.; Burman, C.; Brewster, L. J.; Goitein, M.; Mohan, R. Histogram reduction method for calculating complication probabilities for three-dimensional treatment planning evaluations. Int. J. Radiat. Oncol. Biol. Phys. 21:137-146; 1991.

13. Marks, J. E.; Bedwinek, J. M.; Lee, F.; Purdy, J. A.; Perez, 
C. A. Dose-response analysis for nasopharyngeal carcinoma. Cancer 50:1042-1050; 1982.

14. Miralbell, R.; Crowell, C.; Suit, H. D. Potential improvement of three dimension treatment planning and proton therapy in the outcome of maxillary sinus cancer. Int. J. Radiat. Oncol. Biol. Phys. 22:305-310; 1992.

15. Munzenrider, J. E.; Brown, A. P.; Chu, J. C. H.; Coia, L. R.; Doppke, K. P.; Emami, B.; Kutcher, G. J.; Mohan, R.; Purdy, J. A.; Shank, B.; Simpson, J. R.; Solin, L. J.; Urie, M. M. Numerical scoring of treatment plans. Int. J. Radiat. Oncol. Biol. Phys. 21:147-163; 1991.

16. Niel, C. G. J. H. A reference frame designed to use external numeric references during simulation for tumours located in the head. Br. J. Radiol. 66:927-929; 1993.

17. Perez, C. A.; Stanley, K. E.; Grundy, G.; Hanson, W.; Rubin, P.; Kramer, S.; Brady, L. W.; Marks, J. E.; PerezTamayo, R.; Brown, G. S.; Concannon, J. P.; Rotman, M. Impact of irradiation technique and tumor extent in tumor control and survival of patients with unresectable non-oat cell carcinoma of the lung. Cancer 50:1091-1099; 1982.

18. Roa, W. H. Y.; Hazuka, M. B.; Sandler, H. M.; Martel, M. K.; Thornton, A. F.; Turrisi, A. T.; Urba, S.; Wolf, G. T.; Lichter, A. S. Results of primary and adjuvant CT-based 3-dimensional radiotherapy for malignant tumors of the paranasal sinuses. Int. J. Radiat. Oncol. Biol. Phys. 28:857$865 ; 1994$.

19. Sakata, K.; Aoki, Y.; Karasawa, K.; Nakagawa, K.; Hase- zawa, K.; Muta, N.; Terahara, A.; Onogi, Y.; Sasaki, Y.; Akanuma, A. Analysis of the results of combined therapy for maxillary carcinoma. Cancer 71:2715-2722; 1993.

20. Sato, Y.; Morita, M.; Takahashi, H.; Watanabe, N.; Kirikae, I. Combined surgery, radiotherapy and regional chemotherapy in carcinoma of the paranasal sinuses. Cancer 25:571579; 1970.

21. Shibuya, H.; Horiuchi, J.; Suzuki, S.; Shioda, S.; Enomoto, S. Maxillary sinus carcinoma: Result of radiation therapy. Int. J. Radiat. Oncol. Biol. Phys. 10:1021-1026; 1984.

22. Thornton, A. F., Jr; Ten Haken, R. K.; Gerhardsson, A.; Correll, M. Three-dimensional motion analysis of an improved head immobilization system for simulation, CT, MRI, and PET imaging. Radiother. Oncol. 20:224-229; 1991.

23. Tsujii, H.; Kamada, T.; Arimoto, T.; Mizoe, J.; Shirato, H.; Matsuoka, Y.; Irie, G. The role of radiotherapy in the management of maxillary sinus carcinoma. Cancer $57: 2261-2266 ; 1986$.

24. Tsujii, H.; Kamada, T.; Matsuoka, Y.; Takamura, A.; Akazawa, T.; Irie, G. The value of treatment planning using CT and an immobilizing shell in radiotherapy for paranasal sinus carcinomas. Int. J. Radiat. Oncol. Biol. Phys. 16:243249; 1989.

25. Verhey, L. J.; Goitein, M.; McNulpy, P.; Munzenrider, J. E.; Suit, H. D. Precise positioning of patients for radiation therapy. Int. J. Radiat. Oncol. Biol. Phys. 8:289-294; 1982. 